\title{
Effect of Channel-Estimation Error on QAM Systems With Antenna Diversity
}

\author{
Bin Xia and Jiangzhou Wang, Senior Member, IEEE
}

\begin{abstract}
This paper studies the effect of channel estimation error and antenna diversity on multilevel quadrature amplitude modulation (M-QAM) systems over Rayleigh fading channels. Based on the characteristic function method, a general closed-form bit-error rate (BER) for M-QAM systems is presented. The effect of the inaccurate channel estimation on the performance for pilot-symbol-assisted modulation M-QAM systems with antenna diversity is investigated. Simulation results for M-QAM ( $M=4,16,64,256$, etc.) show that the analytical method can accurately estimate the system performance. Moreover, numerical results show that with the antenna-diversity technique, the BER performance improves significantly, especially in perfect channel-estimation cases. It is also found that the channelestimation error limits the benefit of antenna diversity. By increasing the length of the channel estimator and the amplitude of the pilot symbol, more accurate channel estimation can be achieved, so that the BER performance is improved.
\end{abstract}

Index Terms-Antenna diversity, channel estimation, multilevel quadrature amplitude modulation (M-QAM), pilot-symbolassisted modulation (PSAM), Rayleigh fading.

\section{INTRODUCTION}

D URING the past decade, multilevel quadrature amplitude modulation (M-QAM) has been considered for high-rate data transmission over wireless links due to its high spectral efficiency [1]-[4]. In [2], the bit-error rate (BER) performance of QAM in additive white Gaussian noise (AWGN) channels shows that with little performance degradation, higher order modulation can be employed to increase the system throughput. However, in a fading environment, M-QAM requires more accurate fading compensation, because the received M-QAM signal suffers from severe amplitude and phase fluctuations, which certainly degrade system performance. Therefore, it is necessary to study the BER performance of M-QAM systems over fading channels.

It is well known that coherent detection in fading channels requires channel-fading information to compensate for amplitude and phase fluctuations, in order to scale the received signal to correspond to the modulation constellation. In many papers concerned with M-QAM systems, channel information is assumed to be perfectly estimated [1], [3], [5], [6]. However, M-QAM

Paper approved by M.-S. Alouini, the Editor for Modulation and Diversity Systems of the IEEE Communications Society. Manuscript received October 7, 2002; revised September 13, 2003; March 6, 2004; and June 3, 2004.

B. Xia was with the Department of Electrical and Electronic Engineering, University of Hong Kong, Hong Kong. He is now with Alcatel Shanghai Bell Co. Ltd., Shanghai, P.R. China.

J. Wang is with the Department of Electrical and Electronic Engineering, University of Hong Kong, Hong Kong (e-mail: jwang@eee.hku.hk).

Digital Object Identifier 10.1109/TCOMM.2005.843423 is more sensitive to amplitude and phase fluctuations than quaternary phase-shift keying (QPSK) or binary phase-shift keying (BPSK) modulation schemes [4]. Channel estimation becomes a key technique to compensate for the fading effect for QAM systems. Recent studies on M-QAM are mostly focused on symbolerror rate (SER) [1], [5], [9]. Some studies on BER performance are mainly based on computer simulations [7], although it provides analytical results for a special case (e.g., perfect channel estimation and two-branch space diversity). The analytical result for the BER of 16/64-QAM in Rayleigh fading with imperfect pilot-symbol-assisted channel estimation was presented by Tang et al. [4], but the integration operation in [4, eq. (40)] requires a heavy computation load, and thus, it is impractical to be extended to diversity cases. So far, no general algorithm exists to accurately provide the analytical BER performance for M-QAM systems in fading channels.

Due to the poor performance of high-order modulations, e.g., 64/256-QAM, in fading channels, especially in low signal-tonoise ratio (SNR) cases, antenna diversity is employed in this paper to improve system performance. Different from space diversity in [7], antenna diversity is achieved by using multiple antennas at the receiver to combat fading without expanding the system bandwidth [8], where antennas are sufficiently separated. By using the characteristic function method [8], an accurate BER calculation for pilot-symbol-assisted modulation (PSAM) M-QAM systems with imperfect channel estimation and different diversity orders in Rayleigh fading channels is obtained.

The paper is organized as follows. Section II presents the system structure, including transmitter, channel, and coherent receiver models. After that, a pilot-symbol-assisted channelestimation algorithm is briefly described. Performance analysis is presented in Section III. In Section IV, comparison and discussion on numerical results with various parameters are given. Finally, some conclusions are drawn in Section V.

\section{SYSTEM MODELS}

The overall diagram of M-QAM systems is shown in Fig. 1, which includes transmitter, channel, and receiver models.

\section{A. Transmitter Model}

As shown in Fig. 1, the data stream in the transmitter is first fed into an M-QAM mapper. At the M-QAM mapper, the data bit stream is split into in-phase (I) and quadrature (Q) bit streams, which are separately Gray coded as $\sqrt{M}$-AM signals and mapped to complex symbols. 


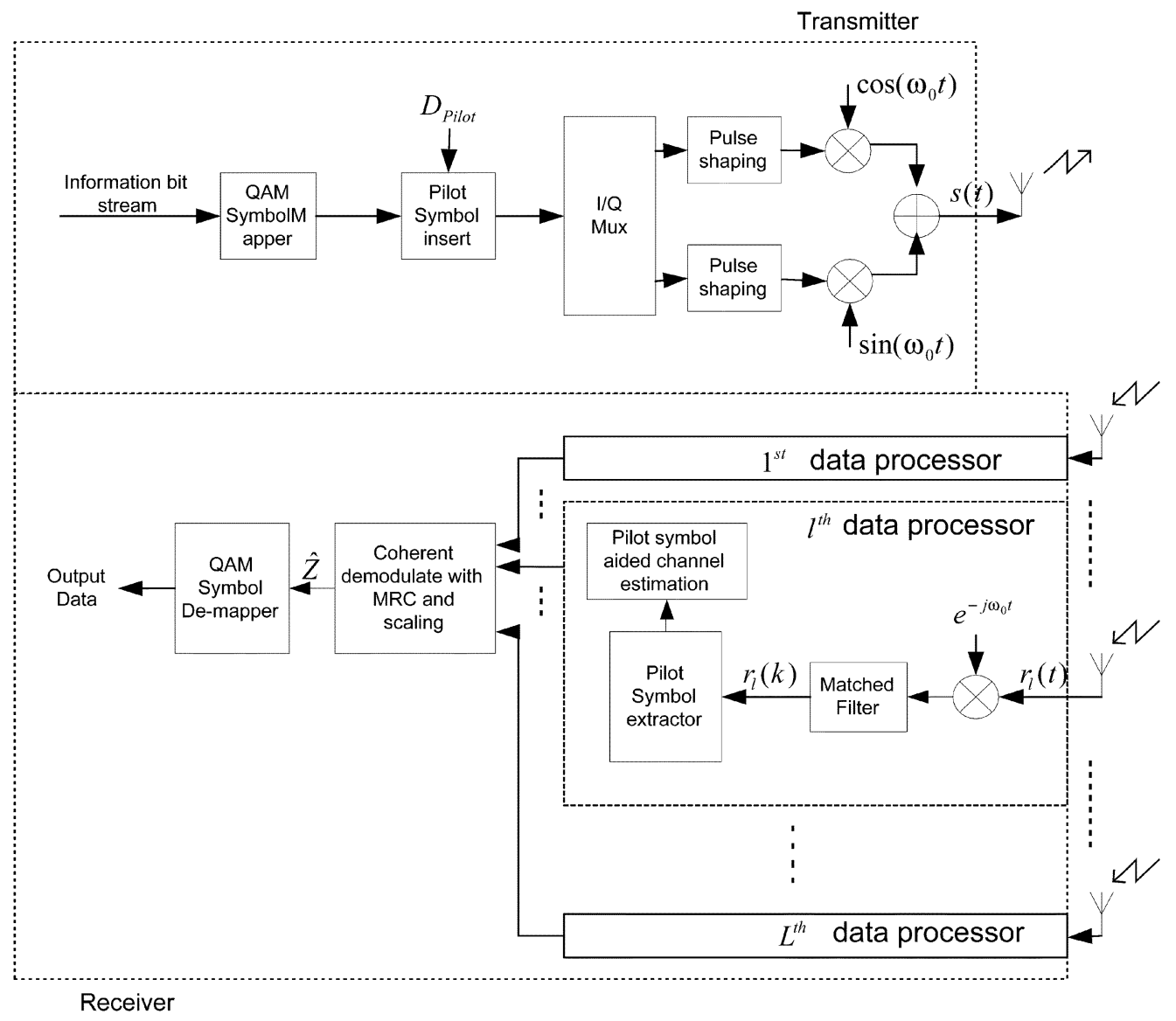

Fig. 1. Block diagram for M-QAM systems.

The I and Q parts of the complex M-QAM symbol are from the set $\{ \pm d, \pm 3 d, \ldots, \pm(\sqrt{M}-1) d\}$, and in order to guarantee equal average energy per bit for fair performance comparison with a different modulation order, $d=\sqrt{\left(3 \log _{2} M / 2(M-1)\right)}$.

The Gray-coding procedure for each $\sqrt{M}$-AM is done as in [3]: sort elements in $\{ \pm d, \pm 3 d, \ldots, \pm(\sqrt{M}-1) d\}$ in ascending order, then label them with integers from 0 to $\sqrt{M-1}$. Finally, convert the integer labels to their binary form. For the $k$ th symbol corresponding to the element $(2 k-1-\sqrt{M}) d$, where $k=1,2, \ldots, \sqrt{M}$, letting its $\left(\log _{2} M / 2\right)$-digit binary equivalent be $b_{1, k} b_{2, k} \ldots b_{\left(\log _{2} M / 2\right), k}$, we obtain $b_{i, k}=\left\lfloor\left(k-1 / 2^{\left(\log _{2} M\right) / 2-i}\right)\right\rfloor \bmod 2$ for $i=1,2, \ldots,\left(\log _{2} M / 2\right)$. Then the corresponding Gray code $g_{1, k} g_{2, k} \ldots g_{\left(\log _{2} M / 2\right), k}$ is given by [3]

$$
\begin{aligned}
g_{1, k} & =b_{1, k} \\
g_{i, k} & =b_{i, k} \oplus b_{i-1, k} \quad i=2,3, \ldots, \frac{\log _{2} M}{2}
\end{aligned}
$$

where $\oplus$ represents modulo- 2 addition. The relationship of the input and output of the encoder is listed in Table I for $M=16$ and 64 with constellations shown in Fig. 2.

In PSAM systems, pilot symbols are periodically inserted. The time-multiplexed pilot symbol $D_{\text {pilot }}$ is known to the receiver. As shown in [4], one pilot symbol is inserted between every $S-1$ data symbols, then an $S$-symbol slot is formatted.

\section{B. Channel Model}

In an $L$-order antenna diversity system, it is assumed that the channel for each receiver antenna is an independent flat-fading channel at a rate much slower than the symbol rate, so the channel remains constant in one symbol duration. For the $l$ th antenna, the phase $\xi_{l}(t)$ is a random variable uniformly distributed in $[0,2 \pi)$, and the fading amplitude $\alpha_{l}(t)$ is a Rayleigh random variable with probability density function (pdf)

$$
p\left(\alpha_{l}(t)\right)=\frac{2 \alpha_{l}(t)}{\Omega_{l}} e^{-\left(\alpha_{l}^{2}(t) / \Omega_{l}\right)}, \quad \alpha_{l}(t)>0
$$

where $\Omega_{l}$ is the average fading power. Assuming equal-gain fading channels, $\Omega_{l}=\Omega$ for all $l$. The autocorrelation of the channel is represented by

$E\left\{\alpha_{l}(t) e^{j \xi_{l}(t)}\left(\alpha_{\hat{l}}(\tau) e^{j \xi_{l}(\tau)}\right)^{*}\right\}=\Omega_{l} J_{0}\left(2 \pi f_{D}(t-\tau)\right) \delta(l-\hat{l})$

where $(x)^{*}$ stands for the conjugation of $x, f_{D}$ is the Doppler frequency of the communication environment, $J_{0}(\cdot)$ is the zeroth-order Bessel function, $\delta(l-\hat{l})$ is equal to one when $l=\hat{l}$, and zero otherwise. $\xi_{l}(t)$ and $\alpha_{l}(t)$ are assumed to be independent for different $l$. 
TABLE I

INPUT/OUTPUT RELATIONSHIP OF GRAY ENCODER FOR M-QAM

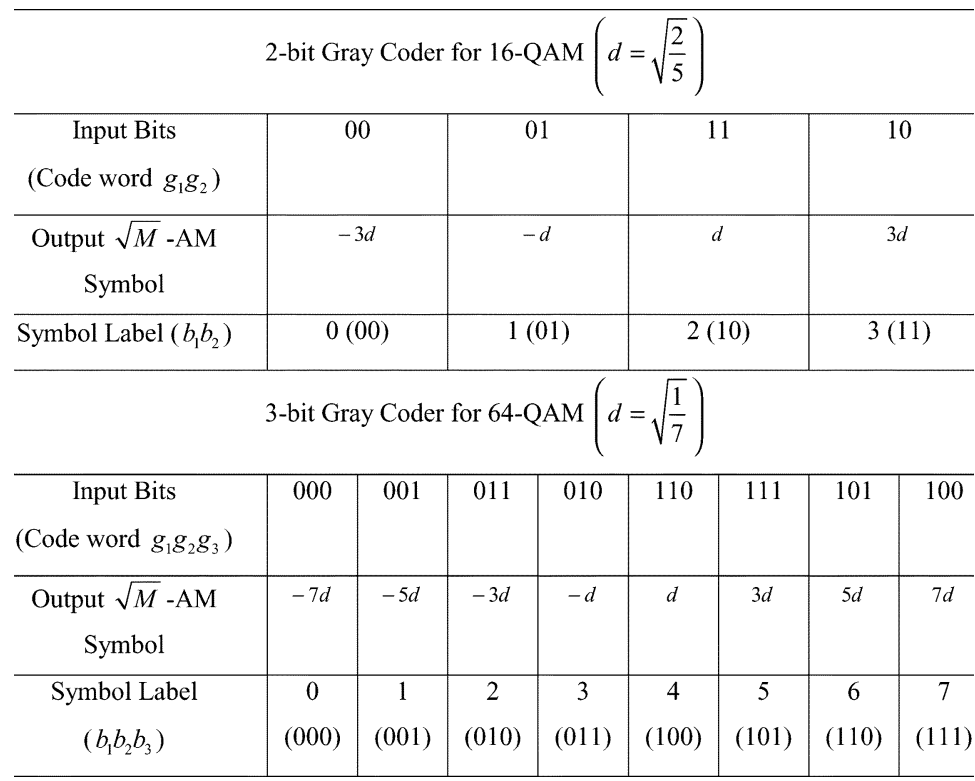

\section{Coherent Receiver Structure}

Assuming perfect carrier and symbol-timing synchronization, the output of the matched filter at the $l$ th antenna for the $m$ th symbol transmitted in the $n$th slot may be written as

$$
\begin{aligned}
r_{l}(n S+m)=\alpha_{l}(n S+m) e^{j \xi_{l}(n S+m)} D & n S+m) \\
& +N_{l}(n S+m)
\end{aligned}
$$

where $D(n S+m)$ is the transmitted QAM symbol, and $N_{l}(n S+m)$ is the background complex AWGN with double-sided power spectral density $\left(N_{0} / 2\right)$.

Without loss of generality, it is assumed that $D(n S)$ is the pilot symbol for $n=0,1, \ldots$. From (4), the desired signal component (the first term on the right-hand side) is perturbed by the fading amplitude $\alpha_{l}(n S+m)$ and phase $\xi_{l}(n S+m)$. If the fading gain or phase is estimated incorrectly, it leads to incorrect M-QAM detection, even in the absence of AWGN [4]. Thus, reliable communication for M-QAM systems requires accurate fading estimation and compensation techniques at the receiver.

In this paper, a coherent multiantenna receiver with pilot-symbol-assisted channel estimation is employed. The pilot-symbol-assisted channel estimator provides estimated fading gain and phase to eliminate the phase rotation, and scale the received signal for data decision. Channel-fading information is extracted by dividing the output of the matched filter for the pilot symbol $r_{l}(n S)$ by the known complex pilot symbol. In PSAM systems, as the fading is time-variant, fading parameters in the pilot-symbol duration are not the same as those in the other data symbols' duration. Moreover, the received signal suffers from AWGN. Thus, channel-estimation error is caused because of time-varying fading and AWGN. Several channel-estimation algorithms have been proposed, including optimal Wiener filter interpolation, low-order Gaussian interpolation (up to second order), or lowpass sinc interpolation. In general, all of them can be regarded as a finite impulse response (FIR) lowpass filter (LPF) with different sets of filter coefficients. Therefore, we will calculate the BER performance with a general FIR channel estimator of $w$ taps. The channel estimation for the $m$ th data symbol transmitted in the $n$th slot duration is given by

$$
\begin{aligned}
\hat{r}_{l}(n S+m)= & \sum_{\text {tap }=-\lfloor(w-1) / 2\rfloor}^{\lfloor w / 2\rfloor} \frac{f_{\text {tap }}^{m} r_{l}((n+\operatorname{tap}) S)}{D_{\text {Pilot }}} \\
= & \sum_{\text {tap }=-\lfloor(w-1) / 2\rfloor}^{\lfloor w / 2\rfloor} f_{\text {tap }}^{m} \alpha_{l}((n+\operatorname{tap}) S) \\
& \times e^{j \xi_{l}((n+\operatorname{tap}) S)} \\
& +\sum_{\text {tap }=-\lfloor(w-1) / 2\rfloor}^{\lfloor w / 2\rfloor} f_{\text {tap }}^{m} \frac{N_{l}((n+\operatorname{tap}) S)}{D_{\text {Pilot }}} .
\end{aligned}
$$

As stated in [4], lowpass sinc interpolation can approach almost the same performance as optimal Wiener interpolation, but with less complexity. But ideal lowpass sinc interpolation is impractical due to its infinite length. Considering the truncation effect of the FIR LPF for channel estimation, the normalized interpolation coefficients of the FIR filter are defined as

$$
f_{\text {tap }}^{m}=\frac{\operatorname{sinc}\left(\frac{m}{S}-\mathrm{tap}\right)}{\sum_{\operatorname{tap}_{1}=-\lfloor(w-1) / 2\rfloor}^{\lfloor w / 2\rfloor} \operatorname{sinc}\left(\frac{m}{S}-\operatorname{tap}_{1}\right)}
$$

where $\operatorname{sinc}(x)=(\sin (\pi x) / \pi x)$.

According to [7], maximal ratio combing (MRC) gives the maximum SNR of the combined signal. Therefore, decision variables for I and Q parts of the $m$ th data symbol transmitted in the $n$th slot duration are

$$
\begin{aligned}
& \hat{Z}^{I}(n S+m)= \\
& \frac{\frac{1}{2} \sum_{l=1}^{L}\left(r_{l}(n S+m) \hat{r}_{l}^{*}(n S+m)+r_{l}^{*}(n S+m) \hat{r}_{l}(n S+m)\right)}{\sum_{l=1}^{L}\left|\hat{r}_{l}(n S+m)\right|^{2}}
\end{aligned}
$$




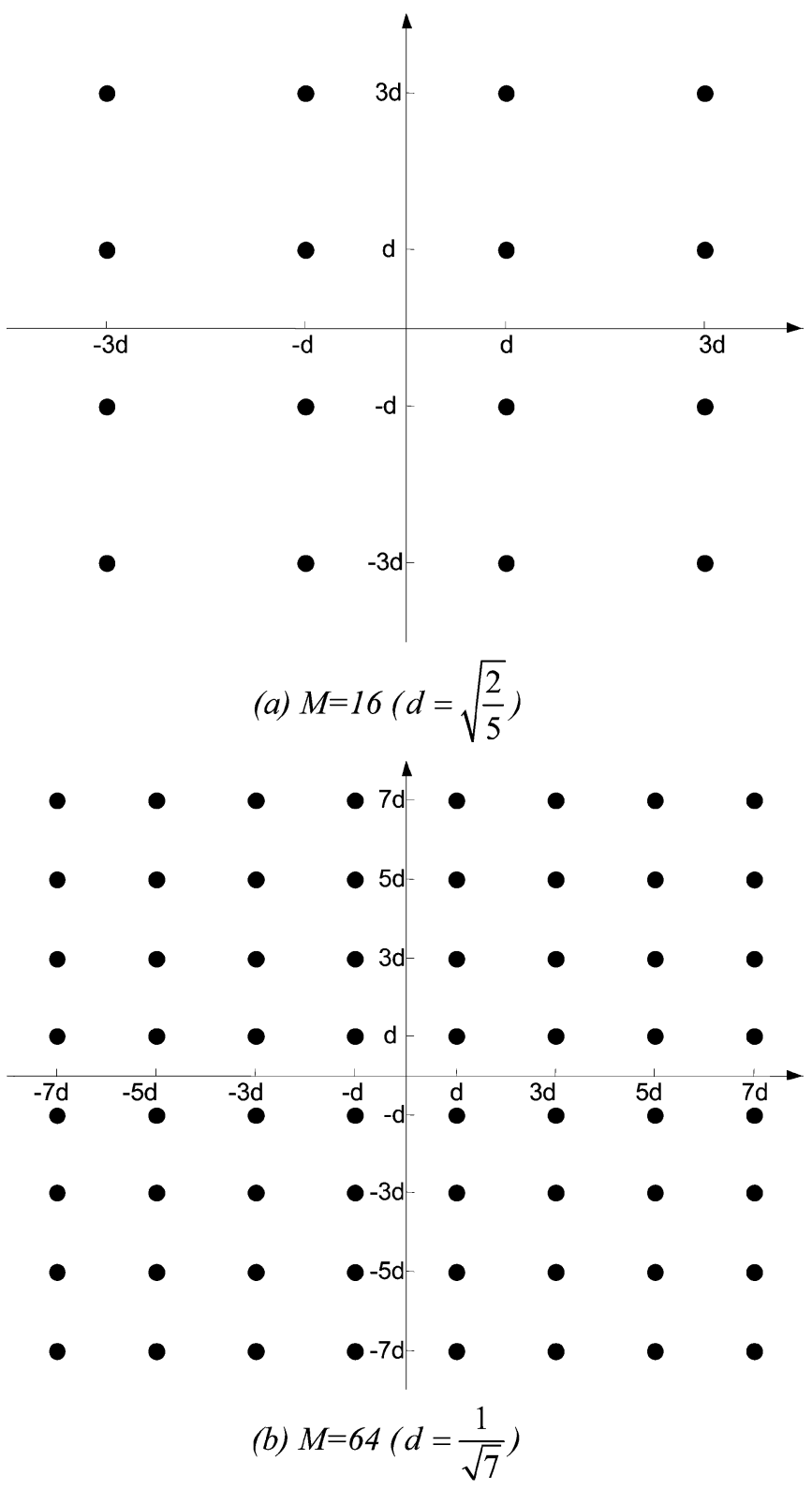

Fig. 2. M-QAM constellation.

and

$$
\begin{aligned}
& \hat{Z}^{Q}(n S+m)= \\
& \frac{\frac{1}{2 j} \sum_{l=1}^{L}\left(r_{l}(n S+m) \hat{r}_{l}^{*}(n S+m)-r_{l}^{*}(n S+m) \hat{r}_{l}(n S+m)\right)}{\sum_{l=1}^{L}\left|\hat{r}_{l}(n S+m)\right|^{2}}
\end{aligned}
$$

respectively. Then I and Q parts of the QAM symbol can be recovered by finding elements in $\{ \pm d, \pm 3 d, \ldots, \pm(\sqrt{M}-1) d\}$, which are closest to $\hat{Z}^{I}(n S+m)$ and $\hat{Z}^{Q}(n S+m)$, respectively.

\section{BER PERFORMANCE ANALYSIS}

\section{A. General BER Performance Formula Derivation}

Since in QAM systems, the outputs of the I and Q channel are symmetric, we will focus on the output of the I channel
$\hat{Z}^{I}(n S+m)$. The BER performance for the $m$ th transmitted symbol in the slot can be obtained by averaging the conditional BER over all possible M-QAM constellation points in the first quadrant, even in the presence of imperfect channel gain and/or phase estimation [4]. Moreover, considering the fact that the quality of channel estimation indicated by the cross-correlation coefficient $\rho$ between $\left|\alpha_{l}(n S+m)\right|^{2}$ and $\left|\hat{r}_{l}(n S+m)\right|^{2}$ [4] may be different for different $m(m=1,2, \ldots, S-1)$, the BER for different $m$ in the slot may be different. Thus, the BER $p_{b}$ for M-QAM is obtained by

$$
p_{b}=\frac{1}{S-1} \sum_{m=1}^{S-1}\left(\frac{4}{M} \sum_{q_{1}=1+\sqrt{M} / 2}^{\sqrt{M}} \sum_{q_{2}=1+\sqrt{M} / 2}^{\sqrt{M}} p_{c, m, q_{1}, q_{2}}\right)
$$

where $p_{c, m, q_{1}, q_{2}}$ is the conditional BER when the transmitted symbol $D(n S+m)$ is $\left(2 q_{1}-1-\sqrt{M}\right) d+$ $j\left(2 q_{2}-1-\sqrt{M}\right) d$.

Concerning the conditional BER $p_{c, m, q_{1}, q_{2}}$ in (9), it can be easily evaluated by averaging over all possibilities of the decision variable $\hat{Z}^{I}(n S+m)$. We can see that for the I part of a given transmitted symbol, i.e., $D^{I}(n S+m)=$ $\left(2 q_{1}-1-\sqrt{M}\right) d$, when $\hat{Z}^{I}(n S+m) \geq(\sqrt{M}-2) d$, the receiver will detect the I part of the transmitted symbol as $(\sqrt{M}-1) d$. Let $H_{k_{1}, k_{2}}$ denote the Hamming distance between code words representing for $\sqrt{M}$-AM symbols $\left(2 k_{1}-1-\sqrt{M}\right) d$ and $\left(2 k_{2}-1-\sqrt{M}\right) d$. Then there is $H_{\sqrt{M}, q_{1}}$-bit error among the $\left(\log _{2} M / 2\right)$ transmitted bits being represented by the I part of the transmitted symbol. For the Gray-coding scheme shown in (1), $H_{k_{1}, k_{2}}$ can be expressed as

$$
H_{k_{1}, k_{2}}=\sum_{i=1}^{\left(\log _{2} M / 2\right)} g_{i, k_{1}} \oplus g_{i, k_{2}}
$$

With the same method to evaluate the rest of the possibilities of $\hat{Z}^{I}(n S+m)$, the conditional error probability $p_{c, m, q_{1}, q_{2}}$ can be written as shown in (11) at the bottom of the next page, where $p_{r}\left(\hat{Z}^{I}(n S+m)<\tilde{B} \mid m, q_{1}, q_{2}\right)$ is the conditional probability of $\hat{Z}^{I}(n S+m)<\tilde{B}$ when the transmitted QAM symbol $D(n S+m)$ is equal to $\left(2 q_{1}-1-\sqrt{M}\right) d+j\left(2 q_{2}-1-\sqrt{M}\right) d$. Therefore, the focus is on calculating the conditional probability of $p_{r}\left(\hat{Z}^{I}(n S+m)<\tilde{B} \mid m, q_{1}, q_{2}\right)$, where $\tilde{B}=\{0, \pm 2 d, \ldots, \pm(\sqrt{M}-2) d\}$ for M-QAM systems. With (7), to calculate the conditional probability of $\hat{Z}^{I}(n S+m)<\tilde{B}$, it is equivalent to calculate the probability of $\sum_{l=1}^{L}\left(-\tilde{B}\left|\hat{r}_{l}(n S+m)\right|^{2}+(1 / 2) r_{l}(n S+m) \hat{r}_{l}^{*}\right.$ $\left.(n S+m)+(1 / 2) r_{l}^{*}(n S+m) \hat{r}_{l}(n S+m)\right)<0$ conditioned on $D(n S+m)=\left(2 q_{1}-1-\sqrt{M}\right) d+j\left(2 q_{2}-1-\sqrt{M}\right) d$. Obviously, it is a special case of the general quadratic form in [8, eq. (B-2)] with $A=0, B=-\tilde{B}$, and $C=1 / 2$. 


\section{B. Conditional BER Performance With Imperfect Channel Estimation}

Applying the characteristic function method in [8, App. B], one obtains

$p_{r}\left(\hat{Z}^{I}(n S+m)<\tilde{B} \mid m, q_{1}, q_{2}\right)=\frac{\sum_{l=0}^{L-1}\left(\begin{array}{c}2 L-1 \\ l\end{array}\right)\left(-\frac{v_{2}}{v_{1}}\right)^{l}}{\left(1-\frac{v_{2}}{v_{1}}\right)^{2 L-1}}$

where

$$
\begin{aligned}
& v_{1}=v_{0}-\sqrt{v_{0}^{2}+\frac{1}{R_{r \mid m, q_{1}, q_{2}} R_{\hat{r} \mid m, q_{1}, q_{2}}-\left|R_{r \hat{r} \mid m, q_{1}, q_{2}}\right|^{2}}} \\
& v_{2}=v_{0}+\sqrt{v_{0}^{2}+\frac{1}{R_{r \mid m, q_{1}, q_{2}} R_{\hat{r} \mid m, q_{1}, q_{2}}-\left|R_{r \hat{r} \mid m, q_{1}, q_{2}}\right|^{2}}}
\end{aligned}
$$

and

$$
v_{0}=\frac{-\tilde{B} R_{\hat{r} \mid m, q_{1}, q_{2}}+\frac{1}{2} R_{r \hat{r} \mid m, q_{1}, q_{2}}^{*}+\frac{1}{2} R_{r \hat{r} \mid m, q_{1}, q_{2}}}{R_{r \mid m, q_{1}, q_{2}} R_{\hat{r} \mid m, q_{1}, q_{2}}-\left|R_{r \hat{r} \mid m, q_{1}, q_{2}}\right|^{2}} .
$$

As to the second-moment functions of the received signal $r_{l}(n S+m)$ and estimated channel information $\hat{r}_{l}(n S+m)$, $R_{r \mid m, q_{1}, q_{2}}, R_{\hat{r} \mid m, q_{1}, q_{2}}$, and $R_{r \hat{r} \mid m, q_{1}, q_{2}}$, they are given by

$$
\begin{aligned}
R_{r \mid m, q_{1}, q_{2}} & \frac{1}{2} E\left\{r_{l}(n S+m) r_{l}^{*}(n S+m) \mid m, q_{1}, q_{2}\right\} \\
= & \frac{\left(\left(2 q_{1}-1-\sqrt{M}\right)^{2}+\left(2 q_{2}-1-\sqrt{M}\right)^{2}\right) d^{2}}{2} \\
& \times\left\{E\left\{\alpha_{l}(n S+m) e^{j \xi_{l}(n S+m)}\left(\alpha_{l}(n S+m) e^{j \xi_{l}(n S+m)}\right)^{*}\right\}\right. \\
& \left.\quad+E\left\{N_{l}(n S+m) N_{l}^{*}(n S+m)\right\}\right\} \\
& \frac{\Omega\left(\left(2 q_{1}-1-\sqrt{M}\right)^{2}+\left(2 q_{2}-1-\sqrt{M}\right)^{2}\right) d^{2}}{2} \\
& +\frac{N_{0}}{2}
\end{aligned}
$$

where the desired signal is uncorrelated with AWGN components, as shown in (17) at the bottom of the page, where

$$
\begin{aligned}
p_{c, m, q_{1}, q_{2}}=\frac{2}{\log _{2} M}\{ & H_{\sqrt{M}, q_{1}} p_{r}\left(\hat{Z}^{I}(n S+m) \geq(\sqrt{M}-2) d \mid m, q_{1}, q_{2}\right) \\
& +\sum_{q=2}^{\sqrt{M}-1} H_{q, q_{1}} p_{r}\left((2 q-2-\sqrt{M}) d \leq \hat{Z}^{I}(n S+m)<(2 q-\sqrt{M}) d \mid m, q_{1}, q_{2}\right) \\
& \left.+H_{1, q_{1}} p_{r}\left(\hat{Z}^{I}(n S+m)<(-\sqrt{M}+2) d \mid m, q_{1}, q_{2}\right)\right\}
\end{aligned}
$$

$$
\begin{aligned}
& R_{\hat{r} \mid m, q_{1}, q_{2}}=\frac{1}{2} E\left\{\hat{r}_{l}(n S+m) \hat{r}_{l}^{*}(n S+m) \mid m, q_{1}, q_{2}\right\} \\
& =\frac{1}{2} E\left\{\sum_{\operatorname{tap} 1=-\lfloor(w-1) / 2\rfloor}^{\lfloor w / 2\rfloor} \sum_{\operatorname{tap} 2=-\lfloor(w-1) / 2\rfloor}^{\lfloor w / 2\rfloor} f_{\operatorname{tap} 1}^{m} f_{\text {tap } 2}^{m} \alpha_{l}((n+\operatorname{tap} 1) S)\right. \\
& \left.\times e^{j \xi_{l}((n+\operatorname{tap} 1) S)}\left(\alpha_{l}((n+\operatorname{tap} 2) S) e^{j \xi_{l}((n+\operatorname{tap} 2) S)}\right)^{*}\right\} \\
& +\frac{1}{2} E\left\{\sum_{\operatorname{tap} 1=-\lfloor(w-1) / 2\rfloor \operatorname{tap} 2=-\lfloor(w-1) / 2\rfloor}^{\lfloor w / 2\rfloor} \sum_{\text {tap } 1}^{\lfloor w / 2\rfloor} f_{\text {tap } 2}^{m} \frac{N_{l}((n+\operatorname{tap} 1) S)}{D_{\text {Pilot }}}\left(\frac{N_{l}((n+\operatorname{tap} 2) S)}{D_{\text {Pilot }}}\right)^{*}\right\} \\
& =\frac{\Omega}{2} \sum_{\operatorname{tap} 1=-\lfloor(w-1) / 2\rfloor \operatorname{tap} 2=-\lfloor(w-1) / 2\rfloor}^{\lfloor w / 2\rfloor} \sum_{\operatorname{tap} 1}^{m} f_{\operatorname{tap} 2}^{m} J_{0}\left(2 \pi f_{D}(\operatorname{tap} 1-\operatorname{tap} 2) S T\right) \\
& +\sum_{\text {tap }=-\lfloor(w-1) / 2\rfloor}^{\lfloor w / 2\rfloor}\left(f_{\text {tap }}^{m}\right)^{2} \frac{N_{0}}{2\left|D_{\text {Pilot }}\right|^{2}}
\end{aligned}
$$


$T$ is the symbol period, $f_{D}$ denotes the Doppler frequency, and

$$
\begin{aligned}
R_{r \hat{r} \mid m, q_{1}, q_{2}} \\
=\frac{1}{2} E\left\{r_{l}(n S+m) \hat{r}_{l}^{*}(n S+m) \mid m, q_{1}, q_{2}\right\} \\
=\frac{\Omega\left(\left(2 q_{1}-1-\sqrt{M}\right) d+j\left(2 q_{2}-1-\sqrt{M}\right) d\right)}{2} \\
\quad \times \sum_{\text {tap }=-\lfloor(w-1) / 2\rfloor}^{\lfloor w / 2\rfloor} f_{\text {tap }}^{m} J_{0}\left(2 \pi f_{D}(m-\operatorname{tap} \cdot S) T\right) .
\end{aligned}
$$

\section{Conditional BER Performance With Perfect Channel Estimation}

To calculate the BER performance with perfect channel estimation, we can assign $\hat{r}_{l}(n S+m)$ with true channel parameters $\alpha_{l}(n S+m) e^{j \xi_{l}(n S+m)}$, so $R_{\hat{r} \mid m, q_{1}, q_{2}}$ and $R_{r \hat{r} \mid m, q_{1}, q_{2}}$ should be replaced with

$$
R_{\hat{r} \mid m, q_{1}, q_{2}}=\frac{1}{2} E\left\{\hat{r}_{l}(n S+m) \hat{r}_{l}^{*}(n S+m) \mid m, q_{1}, q_{2}\right\}=\frac{\Omega}{2}
$$

and

$$
\begin{aligned}
R_{r \hat{r} \mid m, q_{1}, q_{2}} & =\frac{1}{2} E\left\{r_{l}(n S+m) \hat{r}_{l}^{*}(n S+m) \mid m, q_{1}, q_{2}\right\} \\
& =\frac{\Omega D(n S+m)}{2}
\end{aligned}
$$

whereas the other second-moment function $R_{r \mid m, q_{1}, q_{2}}$ is the same as (16).

\section{NumericAl RESUlts}

In this section, we will investigate the effect of different system parameters (such as modulation and diversity orders, the pilot symbol, and the length of FIR filter for channel estimation) on the BER performance of M-QAM systems with perfect and imperfect channel estimation. Unless noted otherwise, the Rayleigh fading power is assumed to be unitary, i.e., $\Omega=1$, pilot symbol $D_{\text {Pilot }}=1+j$, the Doppler frequency $f_{D}$ is $240 \mathrm{~Hz}$ at the symbol transmission rate of $16 k$ symbol/s, i.e., the normalized Doppler spread $f_{D} T=0.015$, the length of the slot $S$ is 16 , the width of the observation window for channel estimation interpolation algorithm $w$ is 15 , and the diversity order $L$ is 2 .

First, we will investigate the BER performance of M-QAM systems without antenna diversity. Fig. 3 shows the BER performance of M-QAM $(M=4,16,64,256)$ versus SNR. To illustrate the accuracy of analytical results, simulation results are plotted in Fig. 3 as well. From the figure, it can be seen that analytical results can accurately predict the system performance. Moreover, the BER performance improves as SNR increases. But as SNR increases, the relative performance degradation due to imperfect channel estimation becomes more serious. This is because in low-SNR cases, the AWGN component seriously corrupts received signals, which results in poor BER performance. Moreover, the quality of channel estimation is very poor, due to the effect of both time-varying fading channel and serious AWGN. Note that the channel-estimation error resulting

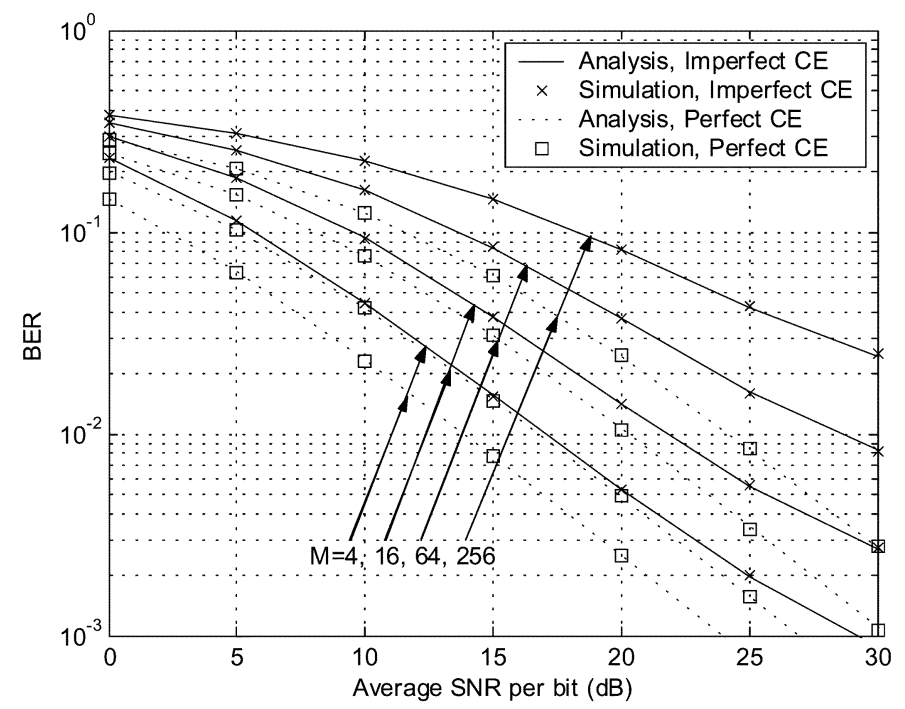

Fig. 3. Analytical and simulation performance of M-QAM without diversity.

from the time-varying fading channel, which is contained in the second line of (5), does not change with different SNR. It becomes dominant to system performance in large-SNR cases, where the AWGN effect is slight. Then the residual crosstalk between I and Q parts of the transmitted data due to inaccurate channel-estimation results in performance degradation. Since the channel-estimation error remains almost constant when the SNR is sufficiently large, performance curves approach error floors. From the figure, it can also be seen that the performance of the 256-QAM system is the worst, even with perfect channel estimation. This is because the constellation map of high-order QAM is more sensitive than that of lower order modulation, i.e., the minimum Euclidean distance of constellation points $(2 d)$ is smaller for higher order modulation. Therefore, for a given SNR, the BER performance degrades as $M$ increases.

Next, we will study the potential of adaptive modulation, i.e., in low-SNR cases, lower order modulation can be employed, whereas in high-SNR cases, at the cost of little BER performance degradation, higher order QAM modulation can be employed to increase system throughput. From Fig. 3, it can be seen that to keep the BER performance at about $10^{-2}$, which is acceptable with the help of a forward error-correcting code (FEC), and with perfect channel estimation, 16-QAM, 64-QAM, and 256-QAM can be adaptively employed when average SNR per bit is greater than 17, 20, and $25 \mathrm{~dB}$, respectively. However, in imperfect channel-estimation cases, extra SNR per bit should be invested, e.g., thresholds for 16-QAM and 64-QAM are at about 23 and $29 \mathrm{~dB}$, respectively. The threshold for 256-QAM is greater than $30 \mathrm{~dB}$, which is somehow impractical due to such a high SNR requirement. Furthermore, the required extra SNR per bit increases as $M$ increases.

As discussed above, in imperfect channel-estimation cases, the performance of 256-QAM is very poor, and 16-QAM and 64-QAM can only be employed in higher SNR cases. To increase system throughput, and also to decrease thresholds for adaptive modulation, antenna diversity is employed. Theoretically, the performance improves with the increase of the diversity order in perfect channel-estimation cases. Here, we 


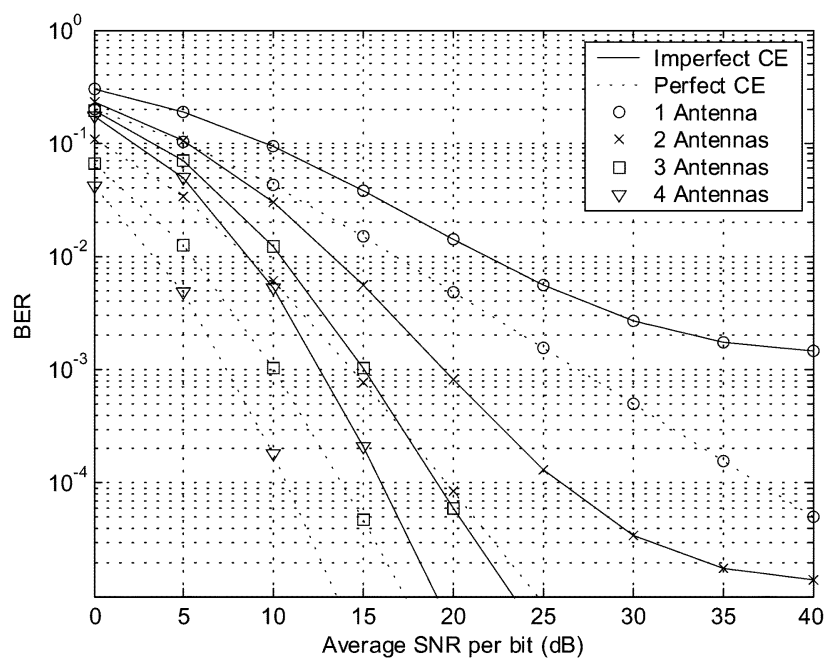

(a) $M=16$

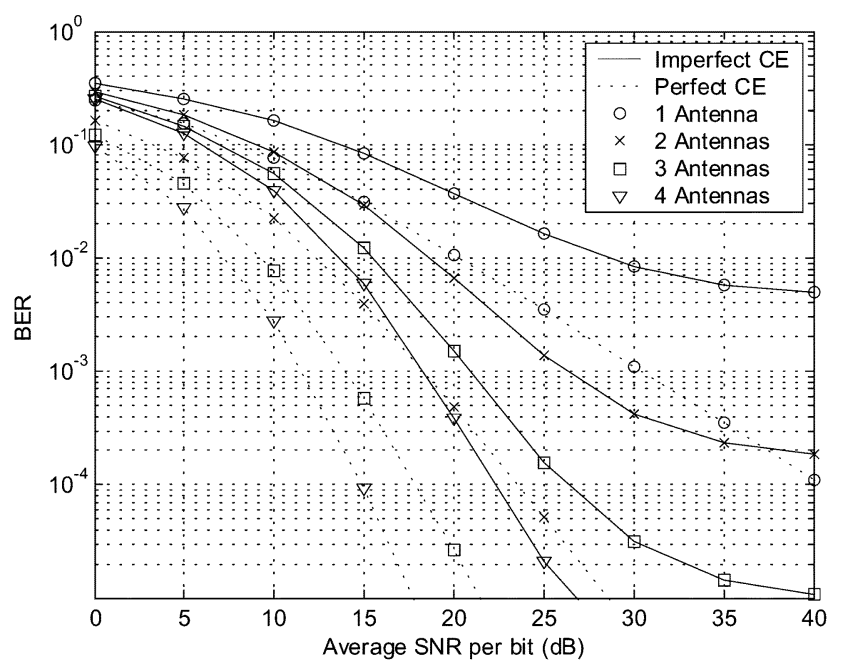

(b) $M=64$

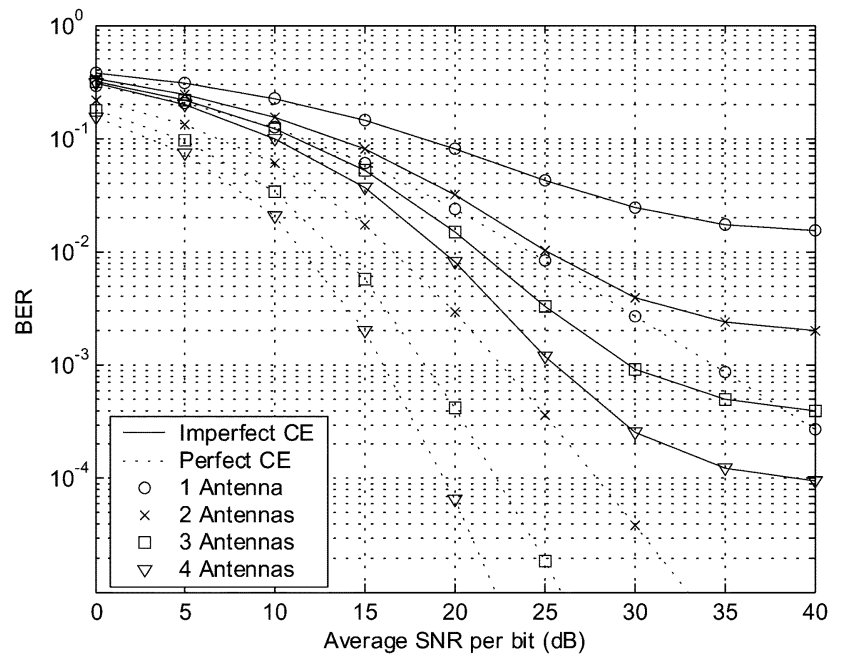

(c) $M=256$

Fig. 4. BER performance of M-QAM with different order antenna diversity.

will investigate the effect of imperfect channel estimation on M-QAM systems with antenna diversity. Fig. 4 illustrates the performance of 16-, 64-, and 256-QAM systems with different diversity orders. It can be seen that the performance is improved rapidly with the increase of the diversity order from one to three, but the improvement becomes slighter when the diversity order is larger. For any given SNR cases, the performance gap between perfect and imperfect channel estimation becomes larger with the increase of diversity order and modulation order, which indicates that inaccurate channel estimation limits the benefit of antenna diversity, especially for higher order modulations. In the case of two antennas, 16-, 64-, and 256-QAM can be adaptively employed in the system when the SNR is greater than 14, 19, and $25 \mathrm{~dB}$, respectively. However, in perfect channel-estimation cases, thresholds significantly decrease to 9,12 , and $16 \mathrm{~dB}$. It implies that if an advanced channel estimator is employed, which can provide more accurate channel estimations, the transmission efficiency can be improved.

Finally, we will study the BER performance of 64-QAM with a different channel-estimation quality. Basically, by increasing the length of the channel estimator, it approaches the ideal lowpass filter with cutoff frequency at $1 / 2 S T$, which can track the time-varying fading well. In other words, increasing the length of the filter means improving the accuracy of the channel estimation. Meanwhile, by increasing the amplitude of the pilot symbol $\left|D_{\text {Pilot }}\right|$, the relative effect of AWGN can be significantly suppressed. Then the channel-estimation quality is improved. Fig. 5 shows the BER performance of 64-QAM versus the length of the channel estimator $w$ with different $\left|D_{\text {Pilot }}\right|$ when the average SNR per bit is $20 \mathrm{~dB}$. It can be seen that the performance improves with the increase of $w$. However, after $w$ increases to a balanced point, to increase $w$ further does not improve the channel-estimation quality significantly, i.e., the second-moment function of channel estimation (17) does not change much in those cases. As a result, the BER reaches error floors. Moreover, by increasing $\left|D_{\text {Pilot }}\right|$, the performance improves due to significant suppression of the AWGN component with respect to pilot components. From the figure, we can find that optimum $w$ is associated with the pilot-symbol amplitude. However, too larger $\left|D_{\text {Pilot }}\right|$ will decrease the transmission efficiency due to power loss for pilot symbols. Therefore, a tradeoff should be made between the transmission efficiency and BER performance. 


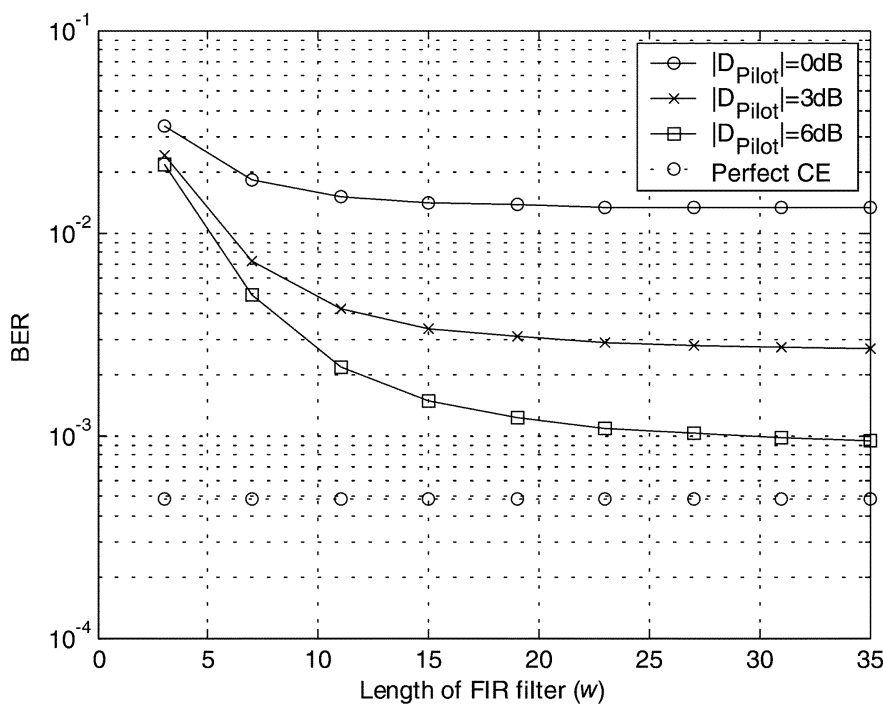

Fig. 5. BER performance of 64-QAM versus length of channel estimator $w$ with different pilot symbols.

\section{CONCLUSION}

In this paper, we have studied the antenna-diversity effect on the BER performance of M-QAM systems in Rayleigh fading channels with imperfect channel estimation. Simulation results show that the BER performance of M-QAM systems can be accurately evaluated by the analytical method in this paper. The main conclusions of this paper can be summarized as follows.

1) QAM is very sensitive to the quality of channel estimation. The performance degradation of higher order QAM is more serious than that of lower order QAM. In the case of perfect channel estimation, to obtain the acceptable BER performance $\left(10^{-2}\right)$, the thresholds for adaptive modulation application of 16-, 64-, and 256-QAM are 17,20 , and $25 \mathrm{~dB}$, respectively. But in the case of imperfect channel estimation, extra SNR per bit should be invested. For higher order modulation, more extra SNR per bit should be invested.

2) Antenna diversity is an efficient technique to improve the BER performance of QAM systems. With the help of antenna diversity, higher order QAM can be employed to improve the system throughput, especially in perfect channel-estimation cases.

3) The channel-estimation error limits the benefit of antenna diversity. If the channel estimator can provide more accurate channel estimation, which approaches perfect channel estimation, system throughput can be increased significantly by using higher order modulation, and the thresholds for adaptive modulation will decrease. Channel estimation can be improved by increasing the length of the channel estimator and the amplitude of the pilot symbol. Both should be determined by balancing the transmission efficiency and BER performance.

\section{ACKNOWLEDGMENT}

The authors would like to thank Dr. M. Sawahashi and Dr. K. Higuchi of NTT DoCoMo for their helpful discussion. The authors also thank the anonymous reviewers for their extremely constructive reviews, which improved the paper significantly.

\section{REFERENCES}

[1] J. Lu, T. T. Tjhung, and C. C. Chai, "Error probability performance of $L$-branch diversity reception of MQAM in Rayleigh fading," IEEE Trans. Commun., vol. 46, pp. 179-181, Feb. 1998.

[2] L. Yang and L. Hanzo, "A recursive algorithm for the error probability evaluation of M-QAM," IEEE Commun. Lett., vol. 4, pp. 304-306, Oct. 2000.

[3] P. K. Vitthaladevuni and M.-S. Alouini, "A recursive algorithm for the exact BER computation of generalized hierarchical QAM constellations," IEEE Trans. Inf. Theory, vol. 49, pp. 297-307, Jan. 2003.

[4] X. Tang, M.-S. Alouini, and A. J. Goldsmith, "Effect of channel estimation error on M-QAM BER performance in Rayleigh fading," IEEE Trans. Commun., vol. 47, pp. 1856-1864, Dec. 1999.

[5] M.-S. Alouini and A. J. Goldsmith, "A unified approach for calculating error rates of linearly modulated signals over generalized fading channels," IEEE Trans. Commun., vol. 47, pp. 1324-1334, Sep. 1999.

[6] A. Annamalai and C. Tellambura, "Error rates for Nakagami- $m$ fading multichannel reception of binary and $M$-ary signals," IEEE Trans. Commun., vol. 49, pp. 58-68, Jan. 2001.

[7] T. Sunaga and S. Sampei, "Performance of multilevel QAM with post detection maximal ratio combining space diversity for digital land-mobile radio communications," IEEE Trans. Veh. Technol., vol. 42, pp. 294-301, Aug. 1993.

[8] J. G. Proakis, Digital Communications. New York: McGraw-Hill, 1995.

[9] S. Sampei and T. Sunaga, "Rayleigh fading compensation for QAM in land mobile radio communications," IEEE Trans. Veh. Technol., vol. 42, pp. 137-147, May 1993.

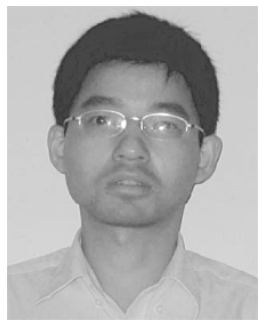

Bin Xia received the B.Eng. degree in electrical engineering and the M.Eng. degree in information and communication engineering from the University of Science and Technology of China, Hefei, China, in 1997 and 2000, respectively, and the Ph.D. degree in electrical engineering from the University of Hong Kong, Kowloon, in 2004.

From 1995 to 2000 , he was with the Personal Communication and Spread Spectrum Laboratory, University of Science and Technology of China, as a Research Engineer involved in the development of CDMA communication systems based on IS-95 and UMTS standards. From 1999 to 2001, he was with UTStarcom Inc., working on WCDMA systems. He is now a System Engineer with Alcatel Shanghai Bell Co. Ltd., Shanghai, P.R. China His research interests are in the areas of broadband wireless-access technologies, wireless networking, and VLSI implementation of wireless transceivers.

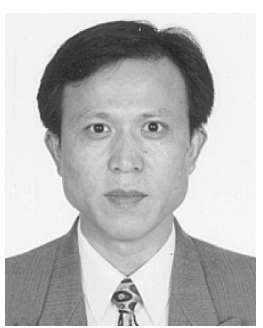

Jiangzhou Wang (M'91-SM'94) received the B.S. and M.S. degrees from Xidian University, Xian, China, in 1983 and 1985, respectively, and the Ph.D. degree (with Greatest Distinction) from the University of Ghent, Belgium, in 1990, all in electrical engineering.

From 1990 to 1992, he was a Postdoctoral Fellow with the University of California, San Diego, CA, where he worked on the research and development of cellular CDMA systems. From 1992 to 1995, he was a Senior System Engineer with Rockwell International Corporation, Newport Beach, CA, where he worked on the development and system design of wireless communications. Since 1995, he has been with the University of Hong Kong, Hong Kong, where he is currently an Associate Professor. He has held a Visiting Professor position with NTT DoCoMo, Japan. He was a Technical Chairman of the IEEE Workshop in 3G Mobile Communications in 2000. He has published over 100 papers, including more than 30 IEEE Transactions/Journal papers in the areas of wireless mobile and spread spectrum communications. He has written/edited two books, Broadband Wireless Communications (Norwell, MA: Kluwer, 2001) and $3 G$ Mobile Enhanced Technologies (Norwood, MA: Artech House, 2002). He holds one U.S. patent in the GSM system. He is listed in Who's Who in the World.

Dr. Wang is an Editor for the IEEE TRANSACTIONS ON COMMUNICATIONS and a Guest Editor for the IEEE Journal ON SELECTED AREAS IN COMMUNICATIONS. 